\title{
INNOVACIÓN EDUCATIVA CON EL USO DE LA GAMIFICACIÓN Y LA ROBÓTICA
}

\section{Serrano-Díaz, N.}

Universidad de Cádiz

noemi.serrano@uca.es

Rioja del Río, C.

Universidad de Cádiz

Cabrera Noguera, E.

Universidad de Cádiz

Fecha de Recepción: 15 Enero 2019

Fecha de Admisión: 30 Abril 2019

\section{RESUMEN}

Motivar e interesar al alumnado en el estudio de las asignaturas STEM (en inglés Science, Technology, Engineering \& Maths) es un nuevo reto para la Unión Europea.

Se presenta un proyecto de investigación H2020 que se está desarrollando actualmente en cinco países europeos (Finlandia, Bielorrusia, Grecia, Alemania y España) y que pretende promover el gusto por el estudio de estas asignaturas entre el alumnado de 10 a 18 años.

El proyecto tiene una duración de 3 años y para establecer los principios pedagógicos se ha llevado a cabo un estudio de corte eminentemente cualitativo a través del análisis de grupos focales, formados por alumnado, profesorado, padres y representantes de empresas del sector.

Se dan a conocer las líneas generales del proyecto, así como la metodología empleada y las actuaciones llevadas a cabo a través de grupos focales y entrevistas a expertos. Se está desarrollando una investigación para conocer las preferencias de los estudiantes al abordar las materias STEM del currículum.

Los resultados, hasta el momento, muestran la necesidad del estudiante de sentir alegría por el estudio y el aprendizaje.

Palabras clave: innovación educativa; STEM; gamificación; robótica

\section{ABSTRACT}

Educational innovation with the use of gamification and robotics. Motivating and interesting students in the study of STEM subjects (in English Science, Technology, Engineering \& Maths) is a new challenge for the European Union.

It presents a research project $\mathrm{H} 2020$ that is currently being developed in five European countries 
(Finland, Belarus, Greece, Germany and Spain) and that aims to promote a taste for the study of these subjects among students from 10 to 18 years.

The project has a duration of 3 years and to establish the pedagogical principles, an eminently qualitative study has been carried out through the analysis of focus groups, formed by students, teachers, parents and representatives of companies in the sector.

The general lines of the project are presented, as well as the methodology used and the actions carried out through focus groups and interviews with experts. An investigation is being developed to know the preferences of the students when approaching the STEM subjects of the curriculum.

The results, so far, show the student's need to feel joy of learning.

Keywords: educational innovation; STEM; gamification; robotics

\section{INTRODUCCIÓN}

Hoy en día la elección de carreras científicas entre los jóvenes está decreciendo. Este creciente desinterés de los jóvenes por las asignaturas STEM (en inglés Science, Technology, Engineering \& Maths) se ha convertido en una preocupación para la Unión Europea por ello "Ciencia con y para la sociedad" (SWAFS) es uno de los objetivos del programa Horizonte 2020.

En este proyecto y relación a las materias STEM, debido a su agrupamiento e interrelación, se trata de desarrollar un tipo de aprendizaje conjunto de las ciencias y las tecnologías, siguiendo el enfoque de Sanders (2009) de una manera conjunta e integrada y no compartimentadas, creando situaciones o problemas para su resolución tal y como se trabaja en Ingeniería (Fortus et al., 2005). Existen hoy en día, probadas evidencias de que esta forma de trabajar las asignaturas da lugar a aprendizajes más efectivos y funcionales (Wai et al., 2010). Para Matacusa et al. (2011) los beneficios son los siguientes:

-Mejora la transferencia de los aprendizajes al mundo real de los estudiantes

-Favorece la retención de los aprendizajes en la memoria a largo plazo

-Incremento de la motivación

Los estudiantes europeos, en general, muestran bajo interés por las carreras tecnológicas y/0 científicas (Valero-Matas, Valero-Oteo \& R-Coca, 2017). Son estas carreras las que aportan a la sociedad los avances que hacen, cada día, la vida más fácil, mejoran la calidad de vida. Las tecnologías se han convertido en conocimiento necesario en la mayoría de los puestos de trabajo (Wang \& Degol, 2013). En relación a estas, la opinión de docentes y estudiantes es coincidente al reconocer la utilidad de las tecnologías en el aprendizaje, para la adquisición de competencias y la comprensión de los contenidos educativos (Edmunds, et al., 2012). Además de contribuir al desarrollo de la capacidad investigadora de los estudiantes (Aguilar y Cuesta, 2009).

Por consiguiente, hay autores que estiman necesario un cambio en los modelos de enseñanza a fin de hacer estas carreras más atractivas con aprendizaje activo, gamificación e incluyendo las tecnologías (Valero-Matas, Valero-Oteo y R-Coca, 2017).

Para Zimmerman (2008) el éxito en el aprendizaje depende, en gran medida, del interés que se despierte en lo jóvenes y de la motivación hacia el objeto de estudio, estos dos factores son fundamentales para promover el esfuerzo que supone hacer las tareas de las distintas asignaturas.

\section{DESCRIPCIÓN, ESTRUCTURA Y ENFOQUE DEL PROYECTO}

Stimey es, como se ha comentado anteriormente, un proyecto de investigación horizonte 2020 para el fomento de las carreras científicas y tecnológicas y para la motivación de los estudiantes, de 10 a 18 años de edad, hacia las asignaturas STEM, todo ello a fin de promover el gusto por el estudio de estas asignaturas y fomentar la elección de carreras ligadas a estas ramas del conocimiento.

Se está desarrollando en cinco países europeos y cuenta con tres años de duración. Los países 
son: Finlandia, Bielorrusia, Alemania Grecia y España y están coordinados por la Universidad de Cádiz.

Este proyecto de investigación trata de conocer las preferencias y gustos que pretende motivar y enseñar de forma lúdica estas asignaturas a los estudiantes, para ello se realiza una indagación de las preferencias de los estudiantes hacia el estudio.

Imagen 1. Elementos del proyecto STIMEY para la consecución de aprendizajes significativos y motivación

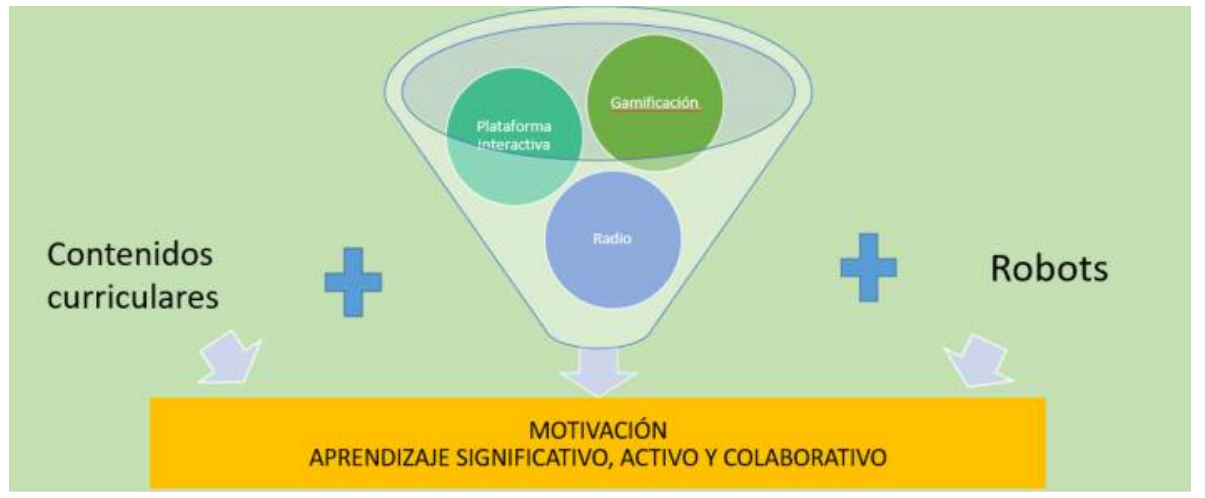

Las estrategias principales propuestas para acercar la ciencia y la tecnología de una manera atractiva que se están creando son:

Una plataforma interactiva en la que el alumnado realizará "misiones" como reto educativo y creativo para llegar a los aprendizajes. Realizando las misiones se consiguen puntos para los centros europeos que participan para conseguir premios. Estas misiones promueven una competitividad sana dado que las actividades se pueden realizar y compartir con otros colegios. Además de las misiones, la plataforma posibilita el aprendizaje el diseño y elaboración de unidades didácticas mediante el empleo de simulaciones y de laboratorios virtuales (Donnelly, et al., 2011).

La plataforma contará también con serious games o juegos pedagógicos con contenido. curricular En esta plataforma tendrán cabida, además del alumnado europeo, los profesores, padres y empresas (empleadores).

Dentro de la plataforma se contempla un espacio para los Mass Media para que los estudiantes europeos puedan chatear, compartir experiencias, conocimientos e inquietudes y puedan trabajar en equipo. Los perfiles podrán ser, además del de estudiantes, de padres y empresas del sector. Esto facilitará la creación de una comunidad STIMEY arropando el proceso de enseñanza y aprendizaje del estudiante.

Radio a la carta, conectado a la plataforma, en la que los estudiantes podrán seleccionar música de distintos estilos y épocas, clasificada, para que, además de su disfrute, exista un aprendizaje musical. Esta radio constará también con entrevistas a profesores relevantes en las distintas áreas científicas y/o tecnológicas, a alumnos, pequeños podcasts con contenidos curriculares, etc,

Robots. Es el elemento motivador y de apoyo al estudiante. A través de los puntos conseguidos cuando se realizan los retos y se finalizan las misiones, los colegios, en una competición sana, podrán conseguir este robot para uso personal de los estudiantes. Con él se podrán hacer búsquedas de información, aprender idiomas, llevar la agenda de estudio y un sinfín de aplicaciones que 


\section{INNOVACIÓN EDUCATIVA CON EL USO DE LA GAMIFICACIÓN Y LA ROBÓTICA}

apoyarán a los estudiantes en su quehacer diario. Además de hablar y acompañar al estudiante, el robot puede expresar emociones, una de las características que expresaron como deseo los estudiantes en los grupos focales.

\section{METODOLOGÍA}

Desde una perspectiva absolutamente cualitativa se trataba de establecer un marco pedagógico y unos principios de diseño para la plataforma en primer lugar, en la comprensión teórica y empírica más avanzada sobre (a) métodos eficientes de enseñanza y aprendizaje, tareas y tipos de actividad, es decir, sobre las formas de enseñanza y aprendizaje y b) los contenidos de la enseñanza y el aprendizaje, en el contexto de las asignaturas STEM, las competencias clave transversales para el aprendizaje permanente y la enseñanza y el aprendizaje reforzados por las TIC para los grupos de edad destinatarios. En segundo lugar, el marco y los principios de diseño se basan en una comprensión empírica más allá del estado actual de la técnica, derivada de la participación de las partes interesadas y en discusiones de grupos focales con las partes interesadas que también alimentarán la teoría.

Para comenzar se realizó un estudio comparativo entre los currículos de los países participantes de los cursos comprendidos entre los 10 y los 18 años de edad, tanto de los temas STEM como de los temas transversales para así abordarlos también en los contenidos de las misiones de manera transversal.

Una vez realizado el estudio comparativo se procedió a la realización de los primeros grupos focales en los distintos países participantes por grupos de edades. Entre los participantes se encontraban: estudiantes, profesores en formación y en ejercicio, padres y madres, expertos en STEM, responsables políticos y representantes de las empresas más relevantes del sector. El enfoque de diseño participativo (Könings et al., 2014) y las técnicas de los grupos focales (Duarte et al., 2015) se combinaron en las sesiones de co-diseño.

El segundo grupo focal trató de confirmar o ratificar los datos extraídos en el primero para cerciorarse de que los análisis realizados y las decisiones que se iban a tomar en función de esos resultados quedaban ratificados.

Una última indagación consistía en someter al criterio de expertos los resultados de la revisión teórica, el currículum y los resultados de los grupos focales. Con esto quedarían establecidos los principios pedagógicos para el diseño de los materiales.

\section{PROCEDIMIENTO}

El procedimiento llevado a cabo en los grupos focales fue presentado como una lista de deseos a fin de motivar a los estudiantes a la participación e implicación.

Las discusiones de los grupos focales recolectaron los deseos de los participantes relacionados con los principales temas tratados en el proyecto STIMEY: Enseñanza y aprendizaje, materias STEM, habilidades curriculares cruzadas, medios sociales, juegos y gamificación, plataforma digital, radio, robots sociales, género y temas de seguridad. Estos temas fueron presentados a los participantes en diapositivas con imágenes inspiradoras de cada tema. Los deseos de los participantes relacionados con diversos temas se recogieron mediante un formulario en línea con preguntas abiertas. También se registraron las discusiones de los grupos focales mediante grabaciones en video.

El análisis de datos cualitativos debe ser sistemático, seguir una secuencia y un orden (ÁlvarezGayou, 2005). El modelo utilizado para el análisis de los datos de los grupos focales realizados lo hemos desarrollado a lo largo de un proceso que puede concretarse en las siguientes fases (Álvarez-Gayou, 2005; Miles y Huberman, 1994; Rubin y Rubin, 1995): 
Obtener la información

Capturar, transcribir y ordenar la información

Codificar la información

Integrar la información

Estos análisis, junto con la toma de datos recogidos tras el análisis de las discusiones grupales recogidas en las grabaciones, dibujarán el marco del proyecto y los principios pedagógicos que regirán el mismo.

Se ha creado, por tanto, una lista de deseos que recogen en su definición las expresiones y/o conceptos de los participantes en los grupos focales agrupados en las categorías y subcategorías revelados tras el análisis.

\section{RESULTADOS}

Tabla 1. Categoría principal extraída del análisis e los grupos focales de los cinco países participantes

$\begin{array}{ll} & \text { Diversión, diversión, satisfacción de los alumnos, clima } \\ & \text { positivo, buenas relaciones entre compañeros, } \\ \text { relaciones amistosas entre profesores y alumnos, } & \text { efectos positivos de la evaluación/ninguna } \\ \text { Alegría de aprender } & \text { ensecuencia negativa; aprender sólo en la escuela/no } \\ & \text { en casa, respetando el tiempo libre de los alumnos. }\end{array}$

La importancia del deseo que tienen por La alegría de aprender fue evidente en todas las respuestas y común a todos los países participantes.

Las partes interesadas participantes se refirieron a la importancia de disfrutar, satisfacer a los alumnos y divertirse. Algunos participantes también comentaron que la evaluación no debería causar consecuencias negativas, sino promover un clima positivo y alentar a los estudiantes a recordar la conexión de las formas de evaluación con la alegría de aprender. Otros participantes expresaron sus preocupaciones en largas jornadas escolares, tareas y trabajo extracurricular a expensas del tiempo libre de los estudiantes, afectando así su alegría de aprender.

También hubo algunos comentarios relacionados con la importancia de un clima social positivo para el aprendizaje fomentado por las buenas relaciones entre iguales y profesor-estudiante, apoyando así se confirma la pertinencia de estos principios de diseño ya identificados en la revisión bibliográfica.

Respecto a la relación profesor-alumno (Mäkelä \& Helfenstein, 2016), tanto las diversas consideraciones teóricas (Bronfenbrenner, 1979; Dewey, 1916; Vygotsky, 1978) como los estudios empíricos (Gislason, 2010; Piispanen, 2008) consideran que las buenas relaciones profesor-alumno y el apoyo de los profesores son cruciales para el aprendizaje y el bienestar. Las relaciones profesoralumno pueden afectar las emociones de rendimiento de los estudiantes y su desregulación reduce las habilidades cognitivas básicas como las habilidades de función ejecutiva (Pnevmatikos y Trikkaliotis, 2013). También son considerados como componentes socioculturales importantes del compromiso escolar (Linnankylä \& Malin, 2008). Algunos aspectos relacionados con el aprendizaje centrado en el estudiante, también asociados con los resultados positivos de los estudiantes, son la empatía, la relación positiva, la no directividad y el aprendizaje alentador (Cornelius-White, 2007).

\section{CONCLUSIÓN}

Resulta una obviedad la necesidad en Europa de fomentar el estudio por las asignaturas STEM $y$, por ende, las carreras relacionadas con estas para fomentar los avances científicos. 
STIMEY es un proyecto de investigación H2020, que está actualmente indagando la manera de promover el gusto por estas asignaturas realizando una investigación de corte eminentemente cualitativa a fin de profundizar en la raíz del problema y oír las voces de los estudiantes ante las situaciones que se están produciendo en Europa, de desinterés y abandono de carreras de estas áreas de conocimiento.

El resultado más llamativo ha sido el mayoritario deseo expresado por los agentes participantes en los grupos focales de Alemania, Grecia, Bielorrusia, Finlandia y España que apuntan a la necesidad de la alegría por aprender 0 Joy of learning.

Este estudio invita a la reflexión e implicación por parte del profesorado de tratar de presentar los contenidos de estas asignaturas de manera más lúdica y menos monótona o tediosa. No deja de ser un reto creativo que tenemos que afrontar, también, en el diseño del proyecto.

Ante este reto nos planteamos el uso de unas novedosas herramientas para atraer, motivar e implicar en el proceso de enseñanza-aprendizaje, tanto al alumnado como al profesorado.

Los profesores podrán participar en este proyecto diseñando misiones para que los estudiantes, de manera globalizada, puedan aprender los contenidos de las asignaturas STEM.

Nos encontramos en las últimas fases del estudio y en breve se podrán compartir con la comunidad educativa la "lista de deseos" de los estudiantes, profesores, padres y madres y empresas del sector europeos para intentar mejorar el panorama actual en Europa.

\section{REFERENCIAS BIBLIOGRÁFICAS}

Aguiar, M. V. \& Cuesta, H. (2009). Importancia de trabajar las TIC en Educación Infantil a través de Métodos como las WebQuest. Revista de Medios y Educación, 34, 81-94

Álvarez-Gayou, J.L. (2005). Cómo hacer investigación cualitativa. Fundamentos y metodología. México: Paidós.

Bronfenbrenner, U. (1979). The ecology of human development. Cambridge, MA: Harvard University Press.

Dewey, J. (1916). Democracy and Education: an introduction to the philosophy of education. Electronic version by the University of Virginia American Studies Program 2003. Disponible en: http://xroads.virginia.edu/ hyper2/Dewey/TOC.html

Duarte, A., Veloso, L., Marques, J. and Sebastião, J. (2015). Site-specific focus groups: analysing learning spaces in situ. International Journal of Social Research Methodology, vol. 18, no. 4, pp. 381-398.

Cornelius-White, J. D. (2007). Learner-centered teacher-student relationships are effective: A metaanalysis. Review of Educational Research 77, 113-143.

Edmunds, R., Thorpe, M., \& Conole, G. (2012). Student attitudes towards and use of ICT in course study, work and social activity: A technology acceptance model approach. British journal of educational technology, 43(1), 71-84.

Fortus, D., Krajcikb, J., Dershimerb, R. C., Marx, R. W. y Mamlok-Naamand, R. (2005). Designbased science and real-world problem solving. International Journal of Science Education, 27(7), 855-879.

Gislason, N. (2010). Architectural Design and the Learning Environment: a framework for school design Research. Learning Environment Research 13, 127-145.

Könings, K. D., Seidel, T., \& van Merriënboer, J. J. (2014). Participatory design of learning environments: integrating perspectives of students, teachers, and designers. Instructional Science, 42(1), 1-9.

Linnankylä, P. \& Malin, A. (2008). Finnish Students' School Engagement in the Light of PISA 2003, 
Scandinavian Journal of Educational Research, 52(6), 583-602.

Mäkelä, T. and Helfenstein, S. (2016) Developing a conceptual framework for participatory design of psychosocial and physical learning environments. Learning Environments Research, vol. 19, no. 3, pp. 411-440.

Mastascusa, E., Snyder, W.J. y Hoyt, B.S. (2011). Effective instruction for STEM disciplines. From learning theory to college teaching. San Francisco: Jossey-Bass.

Miles, M. B. y Huberman, A.M. (1994) Qualitative data analysis: An expanded sourcebook (2a ed.). Thousand Oaks, CA: Sage.

Pnevmatikos, D., \& Trikkaliotis, I. (2013). Intraindividual differences in executive functions during childhood: The role of emotions. Journal of experimental child psychology, 115(2), 245-261.

Piispanen, M. (2008). Good Learning Environment. Perceptions of Good Quality in Comprehensive School by Pupils, Parents and Teachers. University of Jyväskylä. Kokkola University Consortium Chydenius.

Rubin, H.J. y Rubin, I.S. (1995) Qualitative interviewing. The art of hearing data. Thousand Oaks, CA: Sage.

Valero-Matas, J.A., Valero-Oteo, I. \& R-Coca, J. (2017). El Desencuentro entre Ciencia y Educación; Un Problema Científico-Social. International Journal of Sociology of Education,6(3), 296-322. doi: 10.17583/rise.2017.2724

Vygotsky, L. S. (1978). Mind in Society. The Development of Higher Psychological Processes. (M. Cole, Ed.) Cambridge, MA: Harvard University Press.

Wang, M. T., \& Degol, J. (2013). Motivational pathways to STEM career choices: Using expectancy-value perspective to understand individual and gender differences in STEM

fields. Developmental Review, 33(4), 304-340. https://doi.org/10.1016/j.dr.2013.08.001

Wai, J., Lubinski, D., Benbow, C. P., y Steiger J. H.(2010). Accomplishment in science, technology, engineering, and mathematics (STEM) and its relation to STEM educational

Zimmerman, B. J. (2008). Investigating self-regulation and motivation: Historical background, methodological developments, and future prospects. American Educational Research Journal, 45(1), 166-183. dose: A25-year longitudinal study. Journal of Educational Psychology, 102(4), 860-871. 
